\section{Prevalência de sobrepeso e fatores associados em crianças ingressantes no ensino fundamental em um município da região metropolitana de São Paulo, Brasil}

\author{
Overweight, obesity and associated factors in first \\ grade schoolchildren in a city of the metropolitan \\ region of São Paulo, Brazil
}

\author{
${ }^{1}$ Instituto de Saúde, \\ Secretaria de Estado da \\ Saúde de São Paulo, \\ São Paulo, Brasil. \\ 2 Divisão de Alimentação \\ Escolar, Diretoria de \\ Educação de Cajamar \\ Cajamar, Brasil. \\ Correspondência \\ L. Mondini \\ Núcleo de Investigação em \\ Nutrição, Instituto de Saúde \\ Secretaria de Estado da \\ Saúde de São Paulo. \\ Rua Santo Antônio 590, \\ 3o andar, São Paulo. SP \\ 01314-000, Brasil. \\ lmondini@isaude.sp.gov.br
}

\begin{abstract}
This study assessed the prevalence of overweight in public school first graders and the association between overweight and socio-environmental factors in a city in Greater Metropolitan São Paulo, Brazil. Children enrolling in public school first grade in Cajamar in 2005 were assessed. Overweight was defined according to body mass index, by gender and age. Socio-environmental information was obtained using a questionnaire applied to $58.6 \%$ of the children's mothers. Poisson regression models were used to evaluate associations between overweight in schoolchildren and socio-environmental variables. Seventeen percent of the children were overweight. Factors associated with overweight were: mother's obesity ( $P R=3.54$; CI: 2.16-5.80), high consumption of junk food $(P R=2.12$; $C I$ : 1.30-3.45), more than 4 hours a day watching TV ( $P R=2.08 ; C I: 1.03-4.20)$, and daily household per capita availability of vegetal oil (around 3 tablespoons) (PR=1.52; CI: 1.01-2.31). The family environment has a strong influence on overweight in children entering first grade in public schools. Prevention of childhood overweight should preferably target the parents and other caregivers.
\end{abstract}

School Health; Overweight; Motor Activity; Food Consumption

\author{
Lenise Mondini 1 \\ Renata Bertazzi Levy ${ }^{1}$ \\ Silvia Regina Dias Médici Saldiva 1 \\ Sonia Isoyama Venâncio 1 \\ Jeanice de Azevedo Aguiar 2 \\ Maria Lúcia Rosa Stefanini 1
}

\section{Introdução}

A obesidade infantil vem-se constituindo em um dos principais problemas de saúde pública, considerando as evidências de que proporção significativa das crianças obesas torna-se adultos obesos, que a condição de obesidade na infância, persistindo na vida adulta, pode resultar em formas mais severas da obesidade, acompanhadas de elevadas taxas de morbi-mortalidade e que os efeitos negativos da obesidade vêm aumentando na população infantil, especialmente o diabetes mellitus $1,2,3$.

Nos países desenvolvidos tem-se verificado aumento importante na magnitude do sobrepeso e obesidade em crianças e adolescentes. No Canadá, Austrália e parte da Europa as taxas de aumento de excesso de peso na população infantil, na década de 90 , alcançaram $1 \%$ ao ano 4 .

Valores elevados de prevalência da obesidade infantil também são observados nos países em desenvolvimento. No Brasil, no período entre 1974 e 1997, a prevalência de sobrepeso e obesidade mais do que triplicou entre crianças e adolescentes (de 4,1\% para 13,9\%); na China a prevalência de sobrepeso e obesidade entre crianças de seis anos de idade aumentou de 7,7\% para $12,4 \%$ em um período de tempo bastante reduzido, entre 1991 e 1997, e no Chile, entre 1987 e 2000, neste mesmo grupo etário verificou-se prevalência de $12 \%$ para $26 \%$ para os meninos e de $14 \%$ para $27 \%$ em meninas 1 . 
As crianças constituem, portanto, um dos principais grupos-alvo para estratégias de prevenção e controle do sobrepeso e da obesidade, não só devido às suas características como grupo de risco, mas também por conta das possibilidades de sucesso das ações a serem implementadas 1,3 .

A faixa etária entre cinco e sete anos corresponde a uma das fases de maior vulnerabilidade para o desenvolvimento da obesidade. Nesse período, o índice de massa corporal aumenta rapidamente após um período de reduzida adiposidade durante a idade pré-escolar e a "reposição" precoce, rápida e/ou intensa da adiposidade pode indicar aumento do risco de obesidade nos períodos subseqüentes de vida 1,3 .

No período da infância, além de exercer pouco controle sobre o ambiente em que vive, como por exemplo sobre a disponibilidade domiciliar de alimentos, a criança pode ainda sofrer forte influência do hábito alimentar e de atividade física de seus pais e familiares e estar sujeita às mudanças nos padrões ambientais e de comportamento por causa da sua inserção no ambiente escolar 1.

Dessa forma, o ambiente escolar torna-se bastante atrativo para investigar a ocorrência de excesso de peso entre as crianças com idade de seis a sete anos, considerando a cobertura, geralmente elevada, do sistema escolar entre os alunos ingressantes no ensino fundamental e o apoio da rede escolar para a obtenção de informações sobre a criança e sua família, com vistas ao desenvolvimento de programas de prevenção e controle do sobrepeso infantil 1.

Este estudo tem por objetivo conhecer a prevalência de sobrepeso e sua associação com fatores sócio-ambientais em crianças ingressantes na primeira série do ensino público fundamental em um município do Estado de São Paulo, Brasil.

\section{Material e métodos}

Este estudo refere-se à etapa de diagnóstico do projeto Desenvolvimento de Métodos de Educação Nutricional visando à Segurança Alimentar e Nutricional.

\section{População do estudo}

Cajamar é um município com 60.807 habitantes 5, localizado na região metropolitana de São Paulo.

Todos os escolares ingressantes na primeira série do ensino público fundamental, do universo das escolas públicas do Município de Caja- mar, em 2005, foram convidados a participar da pesquisa por meio de carta enviada pela escola aos responsáveis. De um total de 1.154 crianças, $59(5,1 \%)$ não participaram por não retornarem com o termo de consentimento assinado pelo responsável, 40 (3,5\%) tiveram os termos de consentimento não autorizados e 41 (3,5\%) faltaram à escola após duas tentativas adicionais ao primeiro agendamento, sendo, portanto, incluídas no estudo 1.014 crianças.

No município há somente duas escolas particulares, com uma sala de primeira série cada, as quais não foram incluídas no estudo.

O número de crianças ingressantes no ensino fundamental expressa alta cobertura da rede escolar. Dados da Fundação Sistema Estadual de Análise de Dados, no ano de 2005, indicam que o número de crianças com seis anos e sete anos de idade (1.119 e 1.107, respectivamente) é de grandeza semelhante à população do município compatível com idade de ingresso na escola 5 .

\section{Coleta de dados}

A coleta de dados se deu no período entre fevereiro e abril de 2005. As informações tais como data de nascimento e sexo foram obtidas das fichas de matrícula dos alunos.

Os escolares foram pesados e medidos por equipe composta por técnicos de nutrição do município, sob a coordenação de um nutricionista da rede escolar, treinada e padronizada, previamente, pelos autores do estudo.

Para o registro do peso foram utilizadas balanças eletrônicas portáteis da marca Tanita (modelo 1632 de fabricação chinesa) com capacidade para 150kg e precisão de 200 gramas e, para o registro da altura micro-estadiômetros da marca Seca (modelo 208 de fabricação alemã) com $200 \mathrm{~cm}$ e precisão de décimos de centímetros. As medidas foram coletadas e registradas em duplicata sem arredondamentos, sendo a média calculada posteriormente.

O controle de qualidade dos dados antropométricos foi realizado por um nutricionista da equipe do projeto, mediante sorteio de $12 \%$ dos escolares submetidos à segunda medição e pesagem, a fim de detectar possíveis diferenças entre as medidas. Os resultados revelaram valores médios de peso das crianças de $23,29 \mathrm{~kg}$ e de $23,45 \mathrm{~kg}(\mathrm{p}=0,760)$ e valores médios de altura das crianças de $119,58 \mathrm{~cm}$ e de $119,74 \mathrm{~cm}$ ( $\mathrm{p}=0,833$ ) obtidos pela equipe do município e pela equipe de supervisão do projeto, respectivamente.

Uma equipe de profissionais, previamente treinada pelos pesquisadores do projeto foi a campo a fim de coletar informações sobre as condições sócio-econômicas, de saúde, alimen- 
tação e atividade física dos escolares por meio de aplicação de questionário com a mãe, ou responsável pela criança, em entrevistas agendadas nas escolas.

\section{Análise dos dados}

Para a análise do peso e da altura das crianças, foram excluídas quatro crianças, duas delas com defeito físico e duas com idade superior a dez anos. Logo, foram analisados os dados antropométricos de 1.010 crianças distribuídas nas 13 escolas do município, conforme Tabela 1.

Para o diagnóstico do estado nutricional, foi calculado o índice de massa corporal (IMC) pela divisão do peso, em quilos, pelo quadrado da altura, em metros $\left(\mathrm{kg} / \mathrm{m}^{2}\right)$. As crianças foram classificadas com sobrepeso e obesidade considerando os valores de IMC, segundo sexo e idade, propostos por Cole et al. ${ }^{6}$. Adotou-se neste estudo o termo "sobrepeso" para identificar crianças tanto com sobrepeso como com obesidade, constituindo-se na variável desfecho do estudo.

As variáveis demográficas e sócio-ambientais (sexo, idade, escolaridade do chefe da família e das mães, ou responsável pelo cuidado da criança, consumo de alimentos "saudáveis" e "não saudáveis", assistir à TV, disponibilidade familiar per capita de óleo e de açúcar) e o estado nutricional da mãe/responsável da criança constituíram-se nas variáveis independentes do estudo.

A descrição do consumo alimentar dos escolares foi baseada no questionário de freqüência de consumo de treze alimentos na última semana, divididos em dois grupos: alimentos "saudáveis" (frutas, verduras e legumes, leite, feijão, salada verde, sucos naturais), alimentos "não saudáveis" (refrigerantes, salgados fritos, batata frita, sanduíches, salgadinhos de pacote, bolachas/biscoitos, balas/doces/chocolates), utilizado por Castro et al. 7 .

Para a transformação das categorias de freqüência semanal em consumo diário, atribuiu-se a cada um dos alimentos um peso $\left(S_{n}\right)$, de acordo com a categoria de freqüência de consumo: $S_{n}$ $=(1 / 7)[(a+b) / 2]$, em que a e b correspondem aos números de dias da freqüência de consumo na semana, adaptado de Fornés et al. ${ }^{8}$. Às categorias de freqüência "não consome" ou "raramente", atribuiu-se o valor zero. As demais categorias de freqüência semanal foram as seguintes: 1 a 2 vezes; 3 a 4 vezes; 5 a 6 vezes e diariamente (categoria à qual se atribuiu o peso máximo $=1$ ).

A somatória dos valores obtidos para cada alimento constituiu-se em escores de freqüência de consumo para os grupos de alimentos "saudáveis" e "não saudáveis", podendo variar de zero a seis e de zero a sete, respectivamente. A freqüência de
Tabela 1

Distribuição das crianças ingressantes nas primeiras séries das escolas municipais de ensino fundamental. Município de Cajamar, São Paulo, Brasil, 2005.

\begin{tabular}{lcc}
\hline Escolas municipais & Distrito & Crianças \\
\hline Veneranda de F. Pinto & Centro & 130 \\
Maria Elce M. Bertelle & Jordanésia & 135 \\
Antonio Carlos Carvalho & Jordanésia & 125 \\
Maria G. Freitas Gonçalves & Jordanésia & 78 \\
Lucy A. Bertoncini Macias & Jordanésia & 65 \\
Maria de Lourdes Mattar & Polvilho & 114 \\
República do Panamá & Polvilho & 99 \\
Odir Garcia Araújo & Polvilho & 88 \\
Bairro do Paraíso & Polvilho & 51 \\
Bairro do Borelli & Polvilho & 26 \\
Arnaldo C. da Silveira & Rural & 45 \\
Bairro do Gato Preto & Rural & 36 \\
São Benedito & Rural & 18 \\
Total & & 1.010 \\
\hline
\end{tabular}

consumo destes grupos de alimentos foi expressa em tercis, representando o terço de menor freqüência, o terço de freqüência intermediária e o terço de maior freqüência.

A variável "assistir à TV" indicativa de hábito sedentário foi expressa em categorias $(<2$ horas/dia; 2 a 3,99 horas/dia e $\geq 4$ horas/dia) 9 .

A variável "escolaridade" do chefe da família e da mãe ou responsável pelos cuidados da criança foi descrita em categorias de anos de estudo.

As variáveis "disponibilidade per capita diária de óleo" e "disponibilidade per capita diária de açúcar" no domicílio (em litros e quilos, respectivamente) foram obtidas mediante a razão da quantidade comprada destes produtos pelas famílias no último mês e o número de pessoas na família e, posteriormente, dividido por trinta dias.

Para a classificação do estado nutricional da mãe/responsável pela criança, utilizou-se o IMC $\left(\mathrm{kg} / \mathrm{m}^{2}\right)$. Peso e altura foram obtidos por meio dos mesmos equipamentos descritos para a obtenção das medidas antropométricas das crianças. Seguindo as recomendações internacionais, amplamente aceitas, foram classificados com baixo peso, aqueles indivíduos cujo IMC foi inferior a $18,5 \mathrm{~kg} / \mathrm{m}^{2}$, com sobrepeso ou obesidade quando o IMC encontrava-se entre 25,0 e $30,0 \mathrm{~kg} / \mathrm{m}^{2}$ ou IMC igual ou superior a $30 \mathrm{~kg} / \mathrm{m}^{2}$, respectivamente, sendo excluídas as gestantes e nutrizes 10 . 
A análise descritiva dos dados inclui as prevalências de sobrepeso (sobrepeso e obesidade) dos escolares, assim como a freqüência das variáveis sócio-ambientais e do estado nutricional da mãe/responsável.

A prevalência da variável desfecho foi determinada para as categorias das variáveis independentes na análise bivariada, cuja significância foi dada pelo teste de qui-quadrado. $\mathrm{Na}$ análise multivariada, usou-se a regressão de Poisson 11 para a determinação das razões de prevalência e respectivos intervalos de confiança, sendo selecionadas as variáveis independentes que apresentaram valores $\mathrm{p} \leq 0,30$. Permaneceram no modelo final as variáveis com valores $\mathrm{p}<0,05$.

$\mathrm{O}$ processamento e análise de dados foram realizados no programa Stata versão 7.0 (Stata Corporation, College Station, Estados Unidos).

\section{Aspectos éticos}

O presente projeto foi aprovado pelo Comitê de Ética do Instituto de Saúde e está de acordo com a Resolução $n^{\circ}$. 196/96, do Conselho Nacional de Saúde. Os pais ou responsáveis pelas crianças com diagnóstico de obesidade ou de baixo peso receberam comunicação, por escrito, sugerindo o encaminhamento das mesmas às unidades básicas de saúde do município.

\section{Resultados}

A proporção dos escolares ingressantes no ensino fundamental que apresentaram sobrepeso foi de $17 \%$ ( $10,8 \%$ de sobrepeso e $6,2 \%$ de obesidade).

Em função da não-existência de diferenças estatisticamente significativas em relação ao diagnóstico da situação nutricional dos escolares nos diferentes distritos ( $\mathrm{p}=0,706$ para sobrepeso; $\mathrm{p}=0,581$ para obesidade) e do pequeno número de crianças nas três escolas rurais do município, optou-se por reagrupar as escolas de acordo com a localização geográfica em áreas urbana (distritos Central, Jordanésia e Polvilho) e rural.

As informações sobre as variáveis sócio-ambientais foram obtidas para 592 (58,6\%) crianças cujas mães/responsáveis compareceram à entrevista na escola após duas tentativas adicionais ao primeiro agendamento.

As proporções de ausência das mães às entrevistas foram de $37,7 \%$, 43,7\%, $42,8 \%$ e $31,3 \%$ nos diferentes distritos urbanos do município (Centro, Jordanésa e Polvilho) e na área rural, respectivamente, não diferindo entre as regiões ( $\mathrm{p}=0,3365)$.

Verificou-se, também, que a proporção de crianças com excesso de peso cujas mães não compareceram à entrevista não difere daquelas que estavam presentes ( $\mathrm{p}=0,0834$ ).

Na Tabela 2 apresenta-se a distribuição das crianças de acordo com as variáveis demográficas, sócio-ambientais e do estado nutricional da mãe/responsável nas áreas urbana e rural do município.

Chama a atenção, a proporção dos chefes de família com mais de oito anos de estudo na área rural do município que é três vezes menor daquela encontrada na área urbana. As proporções de mães/responsáveis com mais de oito anos de estudo são superiores às do chefe de família, e a diferença entre as áreas rural e urbana persiste, embora em um menor patamar quando comparada às do chefe de família.

A freqüência reduzida de consumo de alimentos "saudáveis" (primeiro tercil) pelos escolares é verificada em mais de $1 / 3$ das crianças na área urbana, enquanto a freqüência elevada (terceiro tercil) de consumo de alimentos "não saudáveis" pelos escolares não difere de forma expressiva entre as áreas urbana e rural.

A proporção de crianças que assistem à TV por mais de duas horas/dia é extremamente elevada, inclusive na área rural.

A disponibilidade média familiar per capita de óleo corresponde aproximadamente a 3,5 colheres de sopa/dia na área urbana do município e de 4,5 colheres de sopa/dia na área rural; a disponibilidade média familiar per capita de açúcar equivale aproximadamente a 4,5 colheres de sopa/dia nas áreas urbanas do município, alcançando 6,5 colheres de sopa/dia na área rural.

O sobrepeso e a obesidade de mães/responsáveis da criança apresentam proporções elevadas no município como um todo; a condição de excesso de peso (sobrepeso e obesidade) ultrapassa $50 \%$ na área urbana e $60 \%$ na área rural.

A Tabela 3 mostra os resultados da análise de regressão bivariada para o sobrepeso das crianças e cada uma das variáveis independentes, sendo excluídas para a construção do modelo final as variáveis "escolaridade do chefe da família" e "disponibilidade média familiar per capita de açúcar", cujo valor de $\mathrm{p}$ foram superiores a 0,30 .

Assim, o modelo final de associação entre o sobrepeso das crianças e as variáveis independentes, elegíveis no modelo bivariado, aponta como "fatores de risco" mães obesas, freqüência elevada de consumo de alimentos "não saudáveis" (corresponde, por exemplo, ao consumo diário de pelo menos dois produtos deste grupo), assistir à televisão diariamente por quatro ou mais horas e a disponibilidade domiciliar per capita de óleo superior a 23,65ml (cerca de três colheres de sopa/dia) (Tabela 4). 
Distribuição (\%) das crianças da primeira série do ensino fundamental de acordo com as variáveis demográficas e sócio-ambientais nas áreas urbana e rural do Município de Cajamar, São Paulo, Brasil, 2005.

\begin{tabular}{|c|c|c|c|c|}
\hline \multirow[t]{2}{*}{ Variáveis } & \multicolumn{2}{|c|}{ Área urbana } & \multicolumn{2}{|c|}{ Área rural } \\
\hline & $\%$ & $\bar{X}(d p)$ & $\%$ & $\bar{X}(d p)$ \\
\hline \multicolumn{5}{|l|}{ Sexo } \\
\hline Masculino & 51,5 & & 50,0 & \\
\hline Feminino & 48,5 & & 50,0 & \\
\hline Idade em meses & & $80,4(4,18)$ & & $81,2(4,85)$ \\
\hline \multicolumn{5}{|l|}{ Escolaridade do chefe da família (anos) } \\
\hline$<4$ & 13,8 & & 26,6 & \\
\hline $4-8$ & 55,0 & & 62,5 & \\
\hline$>8$ & 31,2 & & 10,9 & \\
\hline \multicolumn{5}{|l|}{ Escolaridade da mãe/responsável (anos) } \\
\hline$<4$ & 5,90 & & 8,2 & \\
\hline $4-8$ & 55,1 & & 62,3 & \\
\hline$>8$ & 39,0 & & 29,5 & \\
\hline \multicolumn{5}{|l|}{ Escores de alimentos "saudáveis" } \\
\hline Primeiro tercil $(<2,78)$ & 34,2 & & 29,4 & \\
\hline Segundo tercil $(2,78-3,93)$ & 32,2 & & 35,3 & \\
\hline Terceiro tercil $(>3,93)$ & 33,6 & & 35,3 & \\
\hline \multicolumn{5}{|l|}{ Escores de alimentos "não saudáveis" } \\
\hline Primeiro tercil $(<1,21)$ & 34,9 & & 36,7 & \\
\hline Segundo tercil $(1,21-2,21)$ & 31,7 & & 25,0 & \\
\hline Terceiro tercil $(>2,21)$ & 33,4 & & 38,2 & \\
\hline \multicolumn{5}{|l|}{ Tempo assistindo TV/dia (horas) } \\
\hline$<2$ & 15,6 & & 22,6 & \\
\hline $2-4$ & 48,9 & & 48,4 & \\
\hline$\geq 4$ & 35,5 & & 29,0 & \\
\hline Disponibilidade de óleo per capita/dia (ml) & & $27,1(15,8)$ & & $36,3(20,40)$ \\
\hline Disponibilidade de açúcar per capita/dia (g) & & $55,4(32,2)$ & & $78,2(40,88)$ \\
\hline \multicolumn{5}{|l|}{ Estado nutricional da mãe } \\
\hline Com sobrepeso & 33,2 & & 38,2 & \\
\hline Com obesidade & 19,8 & & 26,5 & \\
\hline
\end{tabular}

Não foram encontradas interações entre as variáveis "alimentos não saudáveis" e "tempo assistindo à televisão", "alimentos não saudáveis" e "disponibilidade domiciliar per capita de óleo" (todos os valores de $\mathrm{p}>0,25$ ).

\section{Discussão}

O excesso de peso em escolares ingressantes nas escolas públicas de primeiro grau do ensino fundamental, crianças com idade aproximada de sete anos, é o principal problema relacionado ao balanço energético (dados não apresentados revelaram prevalência de $0,99 \%$ de baixo peso e de 2,7\% de déficit de estatura) no Município de Cajamar.
Conforme reportado em documento internacional sobre obesidade, as crianças têm mais chance de serem obesas em países ou regiões onde o processo de transição nutricional temse dado rapidamente, apresentando tendência secular positiva do crescimento linear e redução da prevalência do déficit estatural 4 .

No Brasil, este fenômeno tem sido registrado, nas últimas décadas, por meio de estudos com representatividade nacional, e a prevalência de excesso de peso (sobrepeso e obesidade) triplicou entre crianças em idade escolar e adolescentes, enquanto a magnitude do baixo peso foi reduzida, aproximadamente, à metade. Nesse processo, observa-se incremento significativo da altura média das crianças brasileiras aos sete anos de idade; o déficit de estatu- 
Tabela 3

Prevalência (\%) e razão de prevalência (RP) bruta de sobrepeso em crianças ingressantes na primeira série do ensino

fundamental de acordo com as variáveis demográficas e sócio-ambientais. Município de Cajamar, São Paulo, Brasil, 2005.

\begin{tabular}{|c|c|c|c|c|}
\hline Variáveis & $\%$ & RP bruta & IC95\% & $\mathrm{p}$ \\
\hline Área & & & & 0,283 \\
\hline Urbana & 18,1 & 1 & & \\
\hline Rural & 23,5 & 1,3 & $0,81-2,06$ & \\
\hline Sexo & & & & 0,035 \\
\hline Masculino & 15,4 & 1 & & \\
\hline Feminino & 22,2 & 1,44 & $1,02-2,02$ & \\
\hline Idade & & 0,97 & $0,93-1,01$ & 0,241 \\
\hline Escolaridade do chefe da família (anos) & & & & 0,354 \\
\hline$<4$ & 23,0 & 1 & & \\
\hline $4-8$ & 16,9 & 0,73 & $0,46-1,16$ & \\
\hline$>8$ & 20,5 & 0,89 & $0,55-1,45$ & \\
\hline Escolaridade da mãe/responsável (anos) & & & & 0,024 \\
\hline$<4$ & 11,4 & 1 & & \\
\hline $4-8$ & 16,1 & 1,41 & $0,54-3,67$ & \\
\hline$>8$ & 24,6 & 2,15 & $0,83-5,58$ & \\
\hline Escores de alimentos "não saudáveis" & & & & 0,016 \\
\hline Primeiro tercil $(<1,21)$ & 13,6 & 1 & & \\
\hline Segundo tercil $(1,21-2,21)$ & 16,7 & 1,22 & $0,75-1,97$ & \\
\hline Terceiro tercil $(>2,21)$ & 24,4 & 1,79 & $1,16-2,73$ & \\
\hline Escores de alimentos "saudáveis" & & & & 0,068 \\
\hline Primeiro tercil $(<2,78)$ & 21,9 & 1 & & \\
\hline Segundo tercil $(2,78-3,93)$ & 13,2 & 0,59 & $0,38-0,94$ & \\
\hline Terceiro tercil $(>3,93)$ & 19,8 & 0,9 & $0,61-1,33$ & \\
\hline Tempo assistindo TV/dia (horas) & & & & 0,017 \\
\hline$<2$ & 11,1 & 1 & & \\
\hline $2-4$ & 18,3 & 1,64 & $0,83-3.25$ & \\
\hline$\geq 4$ & 25,1 & 2,26 & $1,14-4,47$ & \\
\hline Disponibilidade de óleo domiciliar per capita/dia (ml) & & & & 0,055 \\
\hline$\leq 23,6$ * & 15,5 & 1 & & \\
\hline$>23,6$ & 21,7 & 1,4 & & \\
\hline Disponibilidade de açúcar domiciliar per capita/dia (g) & & & & 0,56 \\
\hline$\leq 52,5$ * & 17,6 & 1 & & \\
\hline$>52,5$ & 19,4 & 1,11 & $0,78-1,56$ & \\
\hline Estado nutricional da mãe & & & & 0 \\
\hline Sem excesso de peso & 11,5 & 1 & & \\
\hline Com sobrepeso & 18,0 & 1,57 & $1,0-2,44$ & \\
\hline Com obesidade & 36,1 & 3,14 & $2,09-4,71$ & \\
\hline
\end{tabular}

* Mediana de disponibilidade domiciliar per capita/dia de óleo e de açúcar.

ra, nessa população, reduziu-se em cerca de $50 \% 4,12,13$.

É interessante relatar que os resultados de um censo de altura de escolares ingressantes na primeira série do primeiro grau no Município de Osasco, São Paulo, parte do qual faz divisa com o Município de Cajamar, realizado há cerca de 15 anos, apontavam quase o dobro da prevalência de déficit de altura entre mesmo grupo etário quando comparada ao município ora estudado 14

Em populações relativamente estáveis, na qual se encontra o Brasil, os fatores ambientais, mais do que os genéticos, podem ter um peso maior na explicação de alterações da prevalência da obesidade ao longo do tempo 4,12 .

Em nosso estudo encontramos como principais fatores independentes associados ao "risco" 
Razão de prevalência (RP) ajustada de sobrepeso em crianças ingressantes na primeira série do ensino fundamental de acordo com as variáveis demográficas e sócio-ambientais. Município de Cajamar, São Paulo, Brasil, 2005

\begin{tabular}{|c|c|c|}
\hline Variáveis & RP ajustada & IC95\% \\
\hline \multicolumn{3}{|l|}{ Estado nutricional da mãe } \\
\hline Sem excesso de peso & 1,0 * & \\
\hline Com sobrepeso & 1,6 & $0,95-2,71$ \\
\hline Com obesidade & 3,54 & $2,16-5,80$ \\
\hline \multicolumn{3}{|c|}{ Escores de alimentos "não saudáveis" } \\
\hline Primeiro tercil $(<1,21)$ & 1,0 * & \\
\hline Segundo tercil $(1,21-2,21)$ & 1,27 & $0,73-2,21$ \\
\hline Terceiro tercil $(>2,21)$ & 2,12 & $1,30-3,45$ \\
\hline \multicolumn{3}{|l|}{ Tempo assistindo TV/dia (horas) } \\
\hline$<2$ & 1,0 * & \\
\hline $2-4$ & 1,3 & $0,64-2,62$ \\
\hline$\geq 4$ & 2,08 & $1,03-4,20$ \\
\hline \multicolumn{3}{|c|}{ Escolaridade da mãe/responsável (anos) } \\
\hline$<4$ & 1 & \\
\hline $4-8$ & 1,03 & $0,37-2,89$ \\
\hline$>8$ & 1,5 & $0,53-4,23$ \\
\hline \multicolumn{3}{|l|}{ Sexo } \\
\hline Masculino & 1 & \\
\hline Feminino & 1,19 & $0,79-1,79$ \\
\hline \multicolumn{3}{|c|}{ Disponibilidade de óleo domiciliar per capita/dia (ml) } \\
\hline$\leq 23,6$ & 1 & \\
\hline$>23,6$ & 1,52 & $1,01-2,31$ \\
\hline \multicolumn{3}{|c|}{ Escores de alimentos "saudáveis" } \\
\hline Primeiro tercil $(<2,78)$ & 1 & \\
\hline Segundo tercil $(2,78-3,93)$ & 0,53 & $0,31-0,90$ \\
\hline Terceiro tercil $(>3,93)$ & 0,87 & $0,55-1,39$ \\
\hline Idade & 0,98 & $0,93-1,03$ \\
\hline \multicolumn{3}{|l|}{ Área } \\
\hline Urbana & 1 & \\
\hline Rural & 1,07 & $0,57-2,00$ \\
\hline
\end{tabular}

* Teste para tendência linear $(p<0,02)$.

de sobrepeso das crianças a condição de obesidade da mãe/responsável da criança, a freqüência elevada de consumo de alimentos "não saudáveis", assistir à televisão por mais de quatro horas/dia e a disponibilidade diária domiciliar per capita de óleo utilizado para cozinhar superior a três colheres de sopa.

Estudos que investigam o aparecimento da obesidade precocemente dão suporte à importância da influência do ambiente familiar sobre o risco de a criança tornar-se obesa, uma vez que a criança sofre grande dependência da decisão dos pais ou familiares. Atitudes da família em relação à compra e apresentação dos alimentos, os hábitos de alimentação e de atividade física e o suporte oferecido para a promoção de atividades de lazer podem influenciar o padrão de alimentação e de atividade física da criança 1,3.

A exemplo disso, achados de estudos realizados no Reino Unido e Estados Unidos com crianças mais novas, com idade entre quatro e seis anos, revelaram que a obesidade dos pais ou o tempo gasto em atividade física vigorosa $\mathrm{e}$ assistindo à televisão estão associados, independentemente, com a adiposidade infantil 9,15.

Não são tão freqüentes na literatura os estudos que analisam a condição nutricional de crianças com idade por volta dos sete anos e de seus fatores associados. Normalmente, entre os estudos disponíveis que contemplam a população infantil estão aqueles que incluem crianças mais velhas, pré-adolescentes ou adolescen- 
tes 16,17,18,19. Com menor disponibilidade, ainda, estão aqueles desenhados apropriadamente para a investigação de causalidade como a coorte desenvolvida no Reino Unido que identificou entre os principais fatores de risco para obesidade em crianças aos sete anos de idade, a obesidade dos pais e o tempo despendido pela criança assistindo à televisão como aqueles com maior força de associação com o desfecho e, ainda, o caso-controle realizado em uma amostra de escolas públicas do Município de São Paulo, com crianças entre 7 e 10 anos de idade, revelando a obesidade dos pais e o tempo assistindo à televisão entre os três principais fatores de risco para a obesidade infantil 20,21.

Resultados derivados do presente estudo são compatíveis com os dos estudos citados acima. Vale considerar, também, que a idade (por volta dos sete anos), associada ao processo de reposição da adiposidade na criança (geralmente entre 5 anos e 7 anos de idade), acaba por contribuir com um tempo de exposição a determinados fatores de risco relativamente curto, podendo minimizar, assim, o efeito da causalidade reversa atribuído aos estudos transversais.

Ressalta-se que a cobertura do sistema escolar, normalmente elevada neste período, associada ao apoio da rede escolar para a geração de informações, sobre a criança e sua família, possibilita a implementação de um modelo de monitoramento do excesso de peso infantil e dos seus principais fatores associados, assim como o de avaliação de políticas e programas desenvolvidos na comunidade em geral e/ou especificamente na comunidade escolar.

Desse modo, associar à tomada de dados antropométricos da criança um pequeno elenco de marcadores do excesso de peso infantil pode ser bastante útil para implantação de um sistema de monitoramento, visto que as recomendações sobre ações preventivas da obesidade apontam para a precocidade em iniciá-las. De fato, resultados de estudos com cortes transversais consecutivos mostraram que a prevalência de sobrepeso e obesidade aumenta entre as crianças mais velhas, devendo-se considerar que além do ambiente familiar poder influenciar fortemente sobre o risco de a criança tornar-se obesa, o ingresso na escola está associado a um período em que se inicia certa independência e a aquisição de novos hábitos 22,23.

A obtenção de informações simples, como o tempo diário que a criança dedica assistindo à televisão é um exemplo de valorização de um sistema de monitoramento sem necessariamente, onerá-lo ou sobrecarregá-lo. No estudo de coorte realizado na Nova Zelândia este item foi identificado como um dos principais fatores de risco pa- ra a obesidade aos sete anos, além de ser preditor do IMC, da capacidade respiratória após exercício físico, do colesterol sérico e do hábito de fumar na idade adulta, sendo atribuído ao excesso do tempo limite (duas horas/dia) assistindo à televisão durante a infância (entre 5 e 15 anos de idade) $17 \%$ de sobrepeso, $15 \%$ da performance física inadequada, $15 \%$ do aumento do colesterol sérico e $17 \%$ do hábito de fumar na idade adulta jovem (aos 26 anos de idade) 24.

A obesidade dos pais também se revelou outro marcador importante da obesidade infantil, apresentando força de associação de magnitude expressiva, variando da ordem de $\mathrm{OR}=2,5$ a OR $=4,25$, quando pai ou mãe é obeso, até $\mathrm{OR}=10,5$ quando ambos são obesos 20,24.

Em relação à alimentação, são poucos os estudos que examinam simultaneamente o crescimento infantil, a adiposidade e o consumo de alimentos de forma longitudinal. Resultados de dois estudos de coorte não encontraram ou encontraram associações fracas entre dieta e o risco de obesidade no início da idade escolar. Em um deles, ao compararem vários padrões de dieta adotados na primeira infância, somente o item identificado como "miscelâneas" apresentou fraca associação com o risco de obesidade aos sete anos 21; em outro, somente as proporções de energia provenientes de gorduras e proteínas, avaliadas entre o segundo e o oitavo ano de vida, explicaram uma pequena variabilidade do IMC das crianças aos oito anos de idade 25 .

Apesar da importância e da "facilidade" de incluir informações sobre a criança e sua família em um sistema de coleta de dados antropométricos em escolas, alguns aspectos operacionais considerados para implantação e desenvolvimento deste estudo devem ser considerados.

O processo de implantação para a coleta de dados no Município de Cajamar contou com o interesse e colaboração da Delegacia de Ensino, que disponibilizou as informações sobre número de escolas públicas e respectivos endereços, de classes, de turnos e de escolares ingressantes nas primeiras séries do ensino fundamental. Com o apoio da Delegacia de Ensino estabeleceu-se o contato com a equipe técnica da área de nutrição e com os diretores das escolas para a apresentação das finalidades do projeto e do grau de participação. O envolvimento dos diretores das escolas e dos professores foi fundamental no processo de apresentação da proposta junto aos pais ou responsáveis pela criança e posterior agendamento das entrevistas. Ainda assim, vale lembrar que, com três tentativas de agendamento, cerca de $40 \%$ dos responsáveis pelas crianças não compareceram às entrevistas nas escolas. 
Embora a elevada proporção de perdas (ausência de mães ou responsáveis pelas crianças) verificada neste estudo não tenha implicado viés de seleção, os gestores devem ter atenção especial a este item em função de possíveis dificuldades para o desenvolvimento de ações de intervenção junto às mães ou responsáveis pelas crianças.

A participação efetiva dos pais é, sem dúvida, um fator relevante no processo de desenvolvimento de um possível sistema de monitoramento, considerando que estudos de intervenções têm mostrado resultados positivos quando há o envolvimento de pelo menos um dos pais da criança em programas para perda e controle de peso infantil, tanto quanto na manutenção dos resultados a médio e longo prazo 1,3,26.

Outras possibilidades de adesão dos responsáveis pelas crianças podem e devem ser discutidas para minimizar a perda de informações e para facilitar o processo de coleta de dados antropométricos como, por exemplo, o envolvimento de professores de educação física, respeitando as diferenças locais.

Outro aspecto importante a ser relatado como produto do projeto foi a possibilidade de promoção da intersetorialidade com as áreas da saúde e de educação. A Secretaria de Saúde foi envolvida no esquema de retaguarda do atendimento dos casos de obesidade diagnosticados e encaminhados às unidades básicas de saúde. Foi discutida com os gestores locais a responsabilidade do setor da saúde sobre a demanda das condições de saúde e nutrição de crianças mais velhas, já que tal responsabilidade costuma estar voltada à população infantil menor de cinco anos de idade, assim como a incorporação na agenda de saúde de outras ações de nutrição e práticas saudáveis de vida, que não aquelas exclusivamente voltadas às deficiências nutricionais. Em relação ao setor educação, este diagnóstico local teve papel relevante na elaboração de projetos pedagógicos, visando à inserção do tema alimentação e nutrição, no currículo escolar.

A proporção de crianças ingressantes no ensino fundamental com sobrepeso é elevada. $\mathrm{O}$ desenvolvimento de medidas de prevenção e controle do excesso de peso neste grupo etário deveria envolver não só a instituição escolar, mas também os pais ou responsáveis pelas crianças, uma vez que o ambiente familiar apresentou forte influência sobre a condição do sobrepeso infantil.

\section{Resumo}

Este estudo verificou a prevalência de sobrepeso e sua associação com fatores sócio-ambientais em crianças ingressantes na primeira série do ensino público fundamental do Município de Cajamar, São Paulo, Brasil, em 2005. Sobrepeso foi definido pelo índice de massa corporal, segundo sexo e idade. As variáveis sócio-ambientais foram obtidas por meio de questionário aplicado junto às mães das crianças (58,6\%). Associação entre sobrepeso e variáveis independentes foi verificada mediante técnica de regressão de Poisson. A prevalência de sobrepeso foi de 17\%. Obesidade materna (RP = 3,54; IC: 2,16-5,80), freqüência elevada de consumo de alimentos "não saudáveis" (RP = 2,12; IC: 1,30-3,45), assistir à televisão por mais de quatro horas/dia $(R P=$ 2,08; IC: 1,03-4,20) e disponibilidade domiciliar per capita de óleo (cerca de três colheres sopa/dia) (RP = 1,52; IC: 1,01-2,31) apresentaram-se como fatores independentes associados ao excesso de peso infantil. $O$ ambiente familiar apresentou forte influência sobre a condição de sobrepeso em escolares ingressantes no ensino fundamental. Ações de prevenção e controle do sobrepeso deveriam envolver a instituição escolar e também os pais ou responsáveis pelas crianças.

Saúde Escolar; Sobrepeso; Atividade Motora; Consumo de Alimentos

\section{Colaboradores}

L. Mondini, R. B. Levy, S. R. D. M. Saldiva, S. I. Venâncio e M. L. R. Stefanini participaram do planejamento do estudo e da redação do artigo. L. Mondini, R. B. Levy, S. R. D. M. Saldiva e J. A. Aguiar participaram da supervisão do trabalho de campo e do controle de qualidade do gerenciamento e análise dos dados.

\section{Agradecimentos}

Ao Conselho Nacional de Desenvolvimento Científico e Tecnológico (processo 503174-2003-3). 


\section{Referências}

1. NSW Centre for Public Health Nutrition. Best options for promoting healthy weight and preventing weight gain in NSW. New South Wales: University of Sidney; 2005.

2. Dietz WH, Gortmaker SL. Preventing obesity in children and adolescents. Annu Rev Public Health 2001; 22:337-53.

3. Gill TP. Key issues in the prevention of obesity. $\mathrm{Br}$ Med Bull 1997; 53:359-88.

4. Lobstein T, Baur L, Uauy R. Obesity in children and young people: a crisis in public health. Obes Rev 2004; 5(1 Suppl):4-85.

5. Fundação Sistema Estadual de Análise de Dados. Informações municipais. http://www.seade.sp.gov. br (acessado em Dez/2005).

6. Cole TJ, Bellizi MC, Flegal KM, Dietz WH. Establishing a standard definition for child overweight and obesity worldwide. BMJ 2000; 320:1240-3.

7. Castro IRR, Cardoso LO, Engstrom EM, Levy-Costa RB, Monteiro CA. Monitoramento de fatores de risco e proteção à saúde do adolescente: descrição de métodos e resultados iniciais da cidade do Rio de Janeiro. São Paulo: Núcleo de Pesquisas Epidemiológicas em Nutrição e Saúde, Universidade de São Paulo; 2004.

8. Fornés NS, Martins IS, Velásquez-Meléndez G, Latorre MRDO. Escores de consumo alimentar e níveis lipêmicos em população de São Paulo, Brasil. Rev Saúde Pública 2002; 36:12-8.

9. Janz KF, Levy SM, Burns TL, Torner JC, Willing MC, Warren JJ. Fatness, physical activity, and television viewing in children during the adiposity rebound period: the Iowa bone development study. Prev Med 2002; 35:563-71.

10. World Health Organization. Obesity: preventing and managing the global epidemic. Geneva: World Health Organization; 1998.

11. Barros AJ, Hirakata VS. Alternatives for logistic regression in cross-sectional studies: an empirical comparison of models that directly estimate the prevalence ratio. BMC Med Res Methodol 2003; 3:21.

12. Wang Y, Monteiro C, Popkin BM. Trends of obesity and underweight in older children and adolescents in the United States, Brazil, China, and Russia. Am J Clin Nutr 2002; 75:971-7.

13. Monteiro CA, Benício MHD, Gouveia NC. Evolução da altura dos brasileiros. In: Monteiro CA, organizador. Velhos e novos males da saúde no Brasil: a evolução do país e de suas doenças. São Paulo: Editora Hucitec/Núcleo de Pesquisas Epidemiológicas em Nutrição e Saúde, Universidade de São Paulo; 1995. p. 126-40.
14. Mondini L, Monteiro CA. A coleta da altura de alunos ingressantes nas escolas de primeiro grau em um sistema de vigilância nutricional: análise dos dados antropométricos. J Pediatr (Rio J) 1994; 70:273-9.

15. Dorosthy AR, Emmett PM, Cowin IS, Reilly JJ. Factors associated with early adiposity rebound. Pediatrics 2000; 105:1115-8.

16. O'Loughlin J, Gray-Donald K, Paradis G, Meshefedjian G. One and two year predictors of excess weight gain among elementary schoolchildren in multiethnic, low-income, inner-city. Am J Epidemiol 2000; 153:739-46.

17. Ekelund U, Sardinha L, Anderssen SA, Harro M, Franks PW, Brage S, et al. Associations between objectively assessed physical activity and indicators of body fatness in 9- to $10 \mathrm{y}$-old European children: a population-based study from 4 distinct regions in Europe (the European Youth Heart Study). Am J Clin Nutr 2004; 80:584-90.

18. Mo-Swan L, Geater AF. Risk factors for childhood obesity in transitional society in Thailand. Int J Obes Relat Metab Disord 1996; 20:697-703.

19. Magalhães VC, Azevedo G, Mendonça S. Prevalência e fatores associados a sobrepeso e obesidade em adolescentes de 15 a 19 anos das regiões Nordeste e Sudeste do Brasil, 1996 e 1997. Cad Saúde Pública 2003; 19 Suppl 1:129-39.

20. Reilly JJ, Armstrong J, Dorosty AR, Emmett PM, Ness A, Rogers I, et al. Early life risk factors for obesity in childhood: cohort study. BMJ 2005; 330:1357-63.

21. Ribeiro IC, Taddei JAAC, Colugnatti F. Obesity among children attending elementary public school in São Paulo, Brazil: a case-control study. Public Health Nutr 2003; 6:659-63.

22. Chinn S, Rona RJ. Prevalence and trends in overweight and obesity in three cross sectional studies of British children, 1974-94. BMJ 2001; 322:24-6.

23. Frye C, Heinrich J. Trends and predictors of overweight and obesity in East German children. Int J Obes 2003; 27:963-9.

24. Hancox RJ, Milne BJ, Poulton R. Association between child and adolescent television viewing and adult health: a longitudinal birth cohort study. Lancet 2004; 364:257-62.

25. Skinner JD, Bounds W, Carruth BR, Morris M, Ziegler P. Predictors of children's body mass index: a longitudinal study of diet and growth in children aged 2-8 y. Int J Obes 2004; 28:476-82.

26. Galal OM, Hulett J. Obesity among schoolchildren in developing countries. Food Nutr Bull 2005; 2 (2 Suppl):261-6.

Recebido em 21/Jun/2006

Versão final reapresentada em 13/Dez/2006

Aprovado em 29/Jan/2007 